\title{
A Remarkable Auroral Event on Jupiter Observed in the Ultraviolet with the Hubble Space Telescope
}

\author{
J. C. Gérard, * D. Grodent, R. Prangé, J. H. Waite, \\ G. R. Gladstone, V. Dols, F. Paresce, A. Storrs, \\ L. Ben Jaffel, K. A. Franke
}

Two sets of ultraviolet images of the Jovian north aurora were obtained with the Faint Object Camera on board the Hubble Space Telescope. The first series shows an intense discrete arc in near corotation with the planet. The maximum apparent molecular hydrogen emission rate corresponds to an electron precipitation of $\sim 1$ watt per square meter, which is about 30,000 times larger than the solar heating by extreme ultraviolet radiation. Such a particle heating rate of the auroral upper atmosphere of Jupiter should cause a large transient temperature increase and generate strong thermospheric winds. Twenty hours after initial observation, the discrete arc had decreased in brightness by more than one order of magnitude. The time scale and magnitude of the change in the ultraviolet aurora leads us to suggest that the discrete Jovian auroral precipitation is related to large-scale variations in the current system, as is the case for Earth's discrete aurorae.

The first oherervations of the ultraviolet (UV) aurora on Jupiter were ohtained by the UV spectrometer (UVS) on hoard the Voyager 1 and 2 spacecraft in $1979(1-3)$. Data accumulated since 1980 with the UV spectrograph on the International Ultravineet Explorer (IUE) satellite (4) have heen used to indirectly characterize the main features of the morphological and brightness distribution of the aurora. From these early ohservations, some information was derived on its remporal behavior. Livengood et al. (4) analyzed 10 years of IUE data and found that the average ohserved auroral hrightness profile was generally stable within a factor of about 2 to 3 . The lack of spatial resolution of these nonimaging instruments did not allow the determination of whether morphological changes (in auroral shape or latitude) were associated with the brightness variations $(4,5)$. Some morphological differences were observed in 1992 on two Hubhle Space Telescope (HST) Faint Object Camera (FOC) images (6) with nearly identical central meridian longitudes separated by about 3 days. However, in this case, hoth the maximum local hrightness and the integrated radiated power showed little variation. The question of temporal variahility is of major importance in under-

J. C. Gerard, D. Grodent, V. Dols, Laboratore de Phy sique Atmosphérique et Planetaire, Institut d'Astrophysique. Université de Liège. Belgium.

R. Prange Institut d'Astrophysique Spatiale and Institut d'Astrophysique de Paris. Université de Paris Sud, Orsay', France.

J. H. Waite, G. F. Gladstone, K. A. Franke, Southwest Research Institute San Antonio, TX, 78228-0570, USA F. Paresce and A. Storrs. Space Telescope Science In stitute, Baltimore, MD 21218, USA.

L. B. Jaftel, Institut d'Astrophysique de Paris. France.

-To whom correspondence should be addressed. standing the origin and the acceleration mechanisms of the auroral particles exciting the Jovian UV aurora.

Basic differences exist between the magnetospheres of Jupiter and the Earth. The Earth's magnerosphere dynamics, controlled by the solar wind dynamo, organize auroral processes in a local time frame of reference with peak activity in the midnight sector. These processes take place on magnetic field lines from the central to distant plasma sheet in the nightside magnetotail. By contrast, the much more extensive Jovian magnetosphere is in quasi-corotation with the planet up to distances of about 20 Jovian radii. This feature explains why the UVS experiment on hoard Voyager (3) did not defect any significant day-night variation

In July 1993, two series of three HST FOC images, each of the UV aurora, were taken nearly 20 hours apart to investigate the question of temporal variahility. The filter isolated a 20-nm-wide region centered on $153 \mathrm{~nm}(7)$, which is dominated by the emission of the molecular hydrogen Lyman hands ( $\mathrm{H}_{2}$ Lyman) and the continuum. The relevant parameters of the observations are specified in Table 1

Unexpectedly, the first series of exposures recorded a very bright auroral event that gave rise to a FOC count level about four times higher than any previous observations made with the same instrumental configuration. Nearly parallel spectrographic measurements made with the IUE (8) reveal that the emitted UV auroral radiation reiched the second highest level recorded in over 12 years of IUE Jovian auroral observations. In the first exposure of the first series (I1) (Fig. 1A), a bright but longitudinally limited portion of the auroral arc is visible near the aprouaching (dawn) limb of the planet. In the third exposure (I3) (Fig. 1B), the hright are is considerahly more extended in longitude. A comparison of the location of the emission morpholugy on the two exposures (9) shows that the entire pattern is in quasi-corotation with the planet. However, the leading edge of the bright are is shifted to smaller longitudes by $\sim 52^{\circ}$, which is less than the $65^{\circ}$ of planetary rotation. This slippage may be catused by a temporal brightness change aleng the auroral oval ower the $107 \mathrm{~min}$ separating the two images.

The quasi-corotation demonstrates the predominantly longitudinal control of the auroral emission of Jupiter already suggested by the first FOC images at the Lyman $\alpha$ frequency, obtained in 1992, (10) and by years of IUE ohservations (4). The aturora has an uncertain brightness distribution along the auroral owal and may have a local time dependence as well as a longitude dependence. We also expect these $\mathrm{H}_{2} \mathrm{Ly}$ man emissions to be considerably brighter near the limb (11). For example, the apparent brigheness near the limb is expected to be about eight times hrighter than an identical aurora would be on the central meridian at a latitude of $60^{\circ} \mathrm{N}$.

In the background-subtracted versions of images 12 and $W 1$ (Fig. 2, A and B, respectively), the bright arc seen in Fig. $1 \mathrm{~A}$ stretches from a latitude of $60^{\circ} \mathrm{N}$ and a system III longitude $\left(\lambda_{111}\right)$ of $180^{\circ}$ to $\sim 70^{\circ} \mathrm{N}$ on the dawn limb. A second, weaker spot of emission extends along the central meridian. Weak, diffuse emission fills the entire polar cap and reaches $-50^{\circ} \mathrm{N}$ near $\lambda_{111}=$ $190^{\circ}$. The brightest pixels correspond to a count rate of $\sim 13$ counts per pixel. This level is higher than previous HST FOC observations $(6,12,13)$, which typically reached 2 to 4 counts per pixel above the disk background for similar exposure times. Converted into an apparent emission rate, the most intense parts of the auroral ares in imiges 11,12 , and 13 reach -3.6 megarayleighs $\left(1 \mathrm{MR}=10^{12}\right.$ photons per square centimeter per second) of $\mathrm{H}_{2}$ Lyman

Table 1. Main characteristics of the HST FOC auroral observations. Abbreviations: UT, universal time; $\lambda$, wavelength; CML, central meridian longitude; NUV, near ultraviolet.

\begin{tabular}{ccccc}
\hline Date & UT & $\begin{array}{c}\text { Central } \\
\lambda(\mathrm{nm})\end{array}$ & $\begin{array}{c}\text { CML } \\
\text { (degrees) }\end{array}$ & Image \\
\hline 17 July & $00: 47$ & 210 & 51 & NUV \\
& $02: 21$ & 153 & 108 & 11 \\
& $03: 48$ & 153 & 150 & 12 \\
& $04: 08$ & 153 & 172 & 13 \\
& $23: 16$ & 153 & 146 & W1 \\
18 July & $00: 41$ & 153 & 198 & W2 \\
& $01: 01$ & 153 & 210 & W3 \\
\hline
\end{tabular}




\section{A Remarkable Auroral Event on Jupiter Observed in the Ultraviolet with the Hubble Space Telescope}

J. C. Gérard, “ D. Grodent, R. Prangé, J. H. Waite,

G. R. Gladstone, V. Dois, F. Paresce, A. Storrs, L. Ben Jaffel, and K. A. Franke 
emission, equivalent to $-6 \mathrm{MR}$ of total $\mathrm{H}_{2}$ emission (14).

Figure 2B illustrates the dramatic change that occurred during about two Jovian rotations. The bright are previously seen at $\lambda_{\text {III }}>180^{\circ}$ faded to a much weaker zone of inhomogeneous emission with a maximum near $60^{\circ} \mathrm{N}, \lambda_{I I I}=200^{\circ}$. Table 2 shows a comparison of the toral power radiated in $\mathrm{H}_{2}$ Lyman in images $\mathrm{I} 2$ and $\mathrm{W} 1$ and the maximum count rates in hoth exposures. The peaks of the auroral emission are in a ratio of nearly 8 to 1 . However, the brightest part of the intense are in Fig. 2A maps into a weak zone of -1 count per pixel, indicating a drop to $1 / 13$ of the local emission rate.

A global view of the auroral morphology and its variation during the event is hest given by composite polar orthographic projections of images $I 1, I 2$, and $I 3$ (Fig. 2C) and $W 1, W 2$, and $W 3$ (Fig. 2I)); they illustrate how the overall decrease in emission affected the morphology of the emission. The bright auroral observed in the first group of images (Fig. 2C) lies close to but not along the oval described by the footprint of the magnetic field lines crossing the cquatorial plane at 30 Jovian radii $\left(R_{1}\right)$. Cerard et al. (12) mapped the aurora observed over a complete Jovian rotation and found that a better fit can he ohtained by shifting the center of the model oval by a few degrees. The leading edge of the bright emission lies near $\lambda_{111}=180^{\circ}$, although a weaker extension is ohserved up to $165^{\circ}$. A secondary weaker alignment is seen along the $160^{\circ}$ meridian, extending from $62^{\circ} \mathrm{N}$ up to the limit of visibility. Dimmer unstructured aurora is visible inside and outside the discrete emission. It reaches an equatorward limit of $-50^{\circ} \mathrm{N}$ at $\lambda_{I I I}=180^{\circ}$ at the $1 \sigma$ threshold applied to the individual images. The corresponding projection 20 hours later (Fig. 2D) shows, in agreement with Fig. 2B, considerable brightness variations, but the morphology is reminiscent of Fig. 2C. A weak emission hand is still present parallel to and outside of the 30 $R_{\mathrm{J}}$ ovil at $\lambda_{11 \mathrm{I}}>180^{\circ}$. These features lead to the conclusion that the bright auroral aro already observed previously in the sector $\lambda_{\text {III }}>180^{\circ}$ overlaid a diffuse auroral region that remained nearly invariant in brightness throughout the event.

Table 2 lists the estimated total radiated power over the half hemisphere facing the Earth derived from both $I 2$ and W1. Using an energy conversion of $\sim 7$ for electron deposition (alssuming that the auroral particles are energetic electrons) in a $\mathrm{H}_{2}$ atmosphere $(15-17)$, we find that the $6 \mathrm{MR}$ of total $H$, emission correspond to a local flux of $\sim 1 \bar{W} \mathrm{~m}^{2}$ and a total radiated power of $10^{12} \mathrm{~W}$. The input of such a high energy flux must considerathly perturb the energy
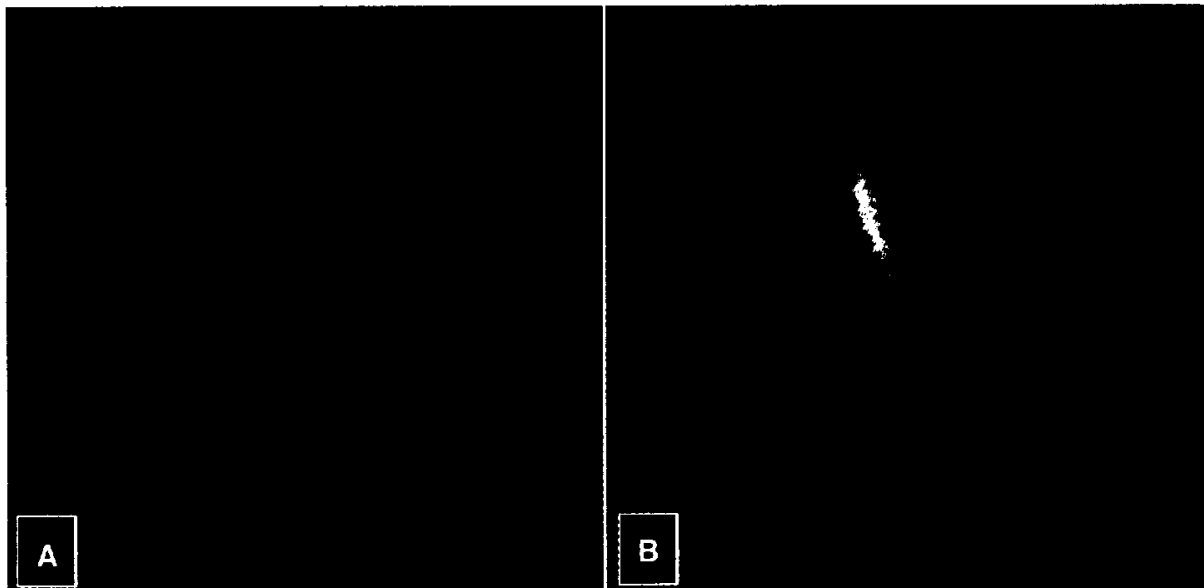

Fig. 1. Images of the Jovian aurora obtained at a wavelength of $153 \mathrm{~nm}$ with the HST FOC. The brightness of the disk background from solar UV radiation scattered by the atmosphere decreases from the mid-latitudes (lower left) to the polar region. The planetary limb is visible against dark space. (A) The first image of the first sequence (exposure |1). The aurora is visible near the limb and forms an ansa or loop near the (astronomical) eastern edge of the oval. (B) The third image (exposure 13) shows how the auroral distribution evolved in the 107 min separating the two exposures.

balance and the temperature profile of the Jovian upper atmosphere. As energetic electrons interact with the hydrogen gas by elastic and inelastic collisions, a latge fraction of the energy input is converted into local gas heating. The consequences of an electron power input of $\sim 1 \mathrm{~W} \mathrm{~m}^{-2}$ can be crudely estimated with simple one-dimensional (1D) energy degradation and energy balance models $(16,18)$.

Because no direct information on the energy distribution of the auroral particles is available, we base our estimates on the HST spectroscopy ohservations of Trafton et al. (19) with a corresponding spectral power index of -4 and a low-energy cutoff at 22 keV. Two situations may be analyzed: (i) A 1D numerical model provides an upper limit of the temperature, and (ii) a more realistic concept that includes the time response and horizontal transport of the 3D atmospheric system is considered. The high-altitude (exospheric) temperature for the case of $1 \mathrm{~W} \mathrm{~m} \mathrm{~m}^{-2}$ calculated at steady state with a $1 D$ model by Waite et al. (16) is $230,000 \mathrm{~K}$, which is much higher than the observed $\mathrm{H}_{3}{ }^{\prime}$ temperature of 1000 to $1500 \mathrm{~K}(20)$. The molecular diffusion times just above the methane homopause where the maximum heating occurs are much longer than a Jovian day. Therefore, we can examine a zonally averaged heating rate, which leads to a reduction in the vertically integrated heat flux $\left(15 \mathrm{ergs} \mathrm{cm}^{-2} \mathrm{~s}^{-1}\right)$ and a corresponding exospheric temperature of $3000 \mathrm{~K}$. The vertical temperature profile for this zonally averaged case is shown in Fig. 3 along with an observationally constrained profile for a more typical aurora (19) (10 to 100 less than the extremely bright aurora of Figs. 1 and 2). The time-variable temperature profile is bounded by these two ex-
Table 2. Local and integrated emission and energy flux.

\begin{tabular}{lcc}
\hline & 12 & $\mathrm{~W} 1$ \\
\hline $\begin{array}{l}\text { Maximum local count } \\
\text { (Counts per pixel) }\end{array}$ & 13.3 & 1.7 \\
$\begin{array}{l}\text { Maximum apparent } \\
\text { emission rate Lyman }\end{array}$ & $>3.6$ & 0.46 \\
$\begin{array}{l}\text { bands and continuum } \\
(\mathrm{MR})\end{array}$ & & \\
$\begin{array}{l}\text { Local energy flux (W m }{ }^{2} \text { ) } \\
\text { Total counts }\end{array}$ & $>0.75$ & 0.1 \\
$\begin{array}{l}\text { Observed radiated power } \\
\left(\mathrm{H}_{2} \text { bands) (1012 W) }\right.\end{array}$ & 18,100 & 5.400 \\
$\begin{array}{l}\text { Estimated precipitated } \\
\text { power (electrons) } \\
\left(10^{12} \mathrm{~W}\right)\end{array}$ & 7 & 0.3 \\
\hline
\end{tabular}

treme cases. The initial temperatures may have risen locally to over $100,000 \mathrm{~K}$. One can speculate that transport subsequently redistributed the heat hy means of advective processes. Finally, a high exospheric temperature of over $3000 \mathrm{~K}$ quite likely persisted for several hours at all auroral longitudes with infrared hot spot structures remaining over the initial zone of heating and significant winds generated as a result of the large energy input.

An additional consideration is the uncertainty in our knowledge of the spectrum of the incoming precipitating particles. The exospheric temperature result is quite sensitive to the low-energy portion of this spectrum hecause the precipitating particles deposit their energy at high altitudes (pressure levels of ahout 1 nbar) that are further removed from the hydrocarton cooling layer. For example, for 215-eV electrons, energy influxes on the order of 100 ergs $\mathrm{cm}^{2}$ $s-1$ produce exospheric temperatures of over $10^{6} \mathrm{~K}$. At these remperatures, the at- 


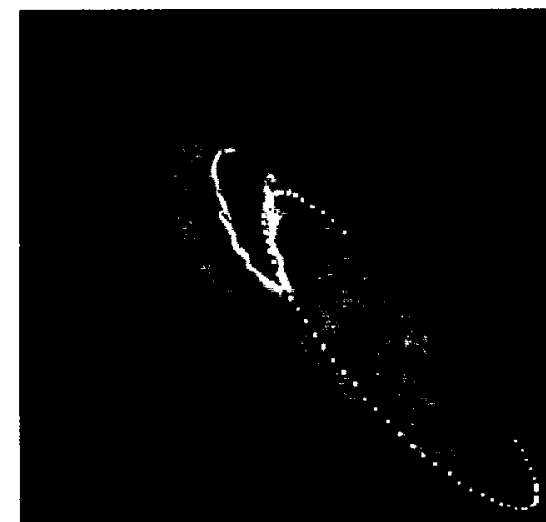

;

A

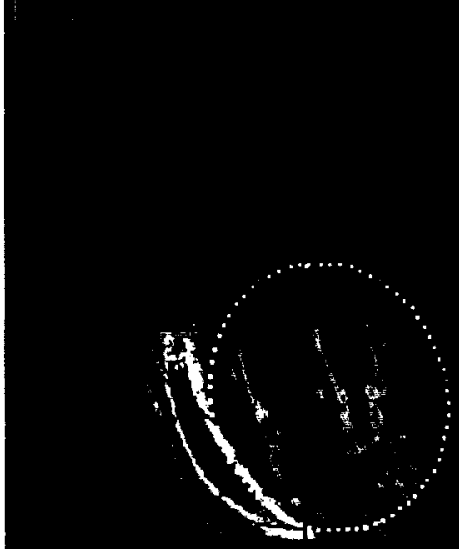

C

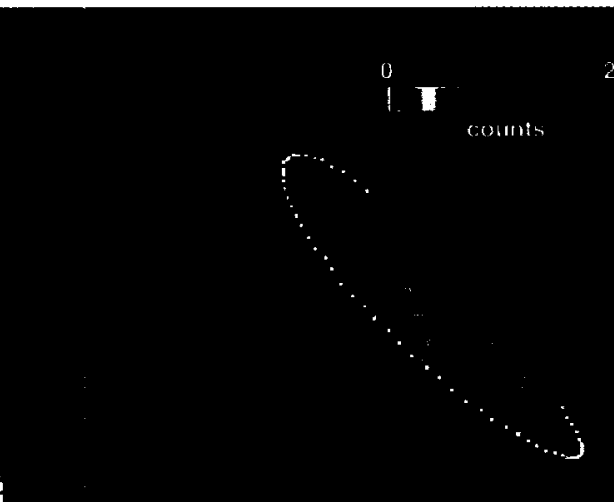

B

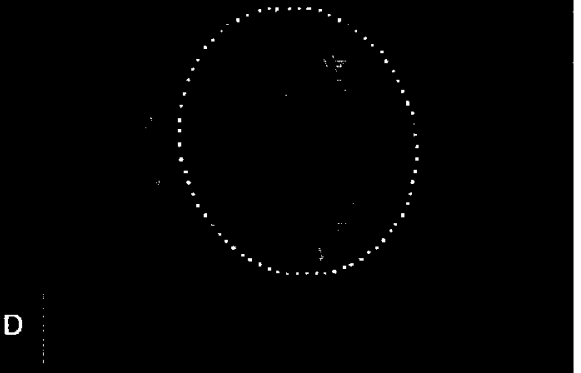

Fig. 2. (A and B) Background-subtracted version of the auroral images with a latitude-longitude grid coordinate (in red). Only features $\geq 1$ a above the background are color-coded. Latitude paralles are separated by $10^{\circ}$ and meridians increase by $20^{\circ}$ from the lower right to the upper left. The white dots indicate the footprint of the $30-R_{\mathrm{J}}$ field lines calculated from the Goddard Space Flight Center (GSFC) $\mathrm{O}_{\text {, }}$ magnetic field model (27). (A) Exposure 12 showing an exceptionally bright aurora with a complex intensity distribution. (B) Exposure $W 1$ showing a dramatic change in auroral brightness over the 19.5 hours since the observation in (A). (C and D) Polar projections of the two sets of bright and weak auroral images where data points $\geq 1 \mathrm{\sigma}$ above the disk background are considered. System III longitudes increase in 20 steps clockwise from the reference meridian $\left(\lambda_{11}-0 \%\right)$, which is oriented toward the top. The $180^{\circ}$ meridian is toward the bottom. (C) A composite of the bright images 11, 12, and 13, and (D) a combination of the weaker images $\mathrm{W}_{1}, \mathrm{~W} 2$, and $\mathrm{W}_{3}$.

mosphere is no longer in hyctrostatic equilibrium and literally hlows off, creating a hydrogen corona. A very high temperature may help explain the highly Doppler-hroadened Lyman a profiles that have been observed by IUE and more recently by HST (21). They mily also explain why the aurord secms to iften accur near the homopatuse level rather than in the thermosphere (where cooling by hydrocirbons is negligihe), particularly at its brightest intensity (22). Indecd, the only stable situation in the case of energy input over 50 ergs $\mathrm{cm}$ $s{ }^{1}$ is when the altitude of the heating and cooling rates nearly coincide.

In the case of the previous exceptional Jowian auroral event ohserved with IUE, it was demonstrated that the auroril enhincement coincided with the arrival at Jupiter of a solar density disturbance, identidied as a coronal mass ejection (5). Therefore, we searched for similar associations with the July 1993 event. The Ulysses spacecraft wats at high heliographic latitude in July 1993 , and the plasma data confirm that it wats nut of the streamer helt at the time of our (Bhservations (23). Therefore, the Ulysses data are not useful in determining the solior wind conditions at Jupiter during the event.

The characteristic time of the observed brightness variation was less than 20 hours. This fictor, together with the auroral lociltion at high latitudes $\left(30 R_{\mathrm{l}}\right)$, indicates that the origin of auroral parricles is not directly connected to the Io plasma torus (6 $\left.R_{1}\right)$. Rather, they appear to originate from the mere distant (middle) magnetesphere and may he linked to field-aligned currents, wherved during the Ulysses encumter with the Jovian magnetesphere, associated with

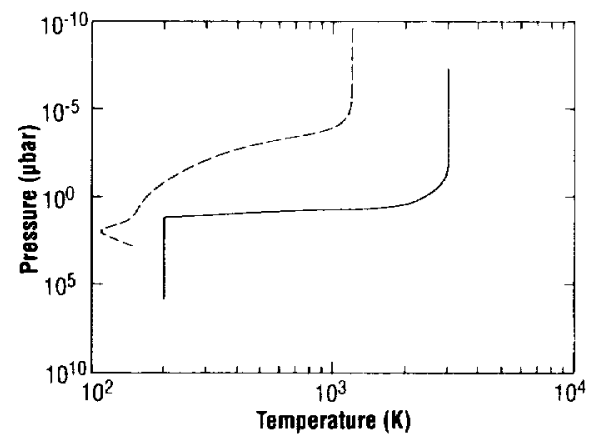

Fig. 3. Steady-state vertical distribution of the temperature of the Jovian auroral atmosphere cal culated for electron precipitation with a $22-\mathrm{keV}$ cutoff and for a total integrated heating rate of 15 ergs $\mathrm{cm}$ " $\mathrm{s}{ }^{\prime}$ (solid line). For comparison, the profile derived from observational spectroscopic constraints (20) is also shown (dotted line).

a high-latitude auroral are ohserved at the Lyman $\alpha$ wavelength with the HST (24). It is interesting to compare the auroral processes at Jupiter with the better decumented and understood terrestrial counterpart. The above arguments (high lititude, short time scale) suggest field-iligned currentdriven auroral precipitations analogous to the terrestrial discrete aurora.

The strongly enhanced auroral emission reported here was ohserved on dayside field lines and was essentially fixed in magnetic longitude, not in locil time. Longitudinally fixed auroral forms are consistent with the dominance of the corotational convective flow within the Jovian magnetosphere. In the inner magnetosphere $\left(<20 R_{1}\right)$, the plasma motion is dominated by the corotattion electric field generated hy the rapid rotation of the Jovian atmosphere-ionosphere. Outside of this distance $\left(20 R_{1}\right)$, the plasma acceleration time becomes longer than the plasma outflow time. This suggests a decoupling of the ionosphere from the magnetosphere, that is, a departure from rigid corotation (25). This presents the possihility that reconnection processes near the magnetupatuse may produce localized solar wind convection cells in the outer magnetosphere, which may result in shears in the plasma fluw near the plasma corotation boundary, in turn producing the fieldaligned currents that are responsible for the high-latitude aurora. Evidence therefore suggests that auroral precipitations, similarly driven on Jupiter and the Earth hy fieldaligned currents, nevertheless originate from different mechanisms.

\section{REFERENCES AND NOTES}

1. A. L. Broadfoot et a., J. Geophys. Res. 86.8250 (1981)

2. B. R. Sandel et al.. Science 206. $962\{1979\}$

3. F. Herbert. B. R. Sandel, A L. Broadfout. I Geo phys Res. 92, 3141 (1987)

A. $T$. A. Livengood. $H$. W. Moos, G. E. Ballesster, 
Prangé, tcarus 97,26 (1992).

5. R. Prangé et al., J. Geophys. Res. 98. 18779(1993)

6. J. C. Gérard, V. Dols, F. Paresce, R. Prange, ibid., p 18793.

7. The overali sensitivity of the optical system is $4.1 \times$ 10 " counts per pixel per second for every kilorayeigh of $\mathrm{H}$., Lyman emission. These observations were made before the addition of the Corrective Optics Space Telescope Axial Replacement (COSTAR) so the aberration of the HST primary mirror spread the light of a point source over a circular halo 2 arc sec in radius. All exposures (896 $\mathrm{s}$ each) described here were made with the use of the same guide star for telescope pointing and tracking. The center of the camera field of view was located along the planel's central meridian at $60 \mathrm{~N}$ during each image

8. F. W. Harris, personal communication.

9. The precise location of the planetary limb in the FOC field of view was determined from both the near-UV exposure (Table 1). which shows no auroral emission, and from the well-defined limb on each 153-rm auroral image.

10. V. Dols. J. C. Gérard, F. Paresce, R. Prangé, A Vidal-Madjar, Geophys. Res. Lett. 19, 1803 (1992).

11. The bulk of the $H$. Lyman emissions are emitted in bands near 160 and $161 \mathrm{~nm}$ and are optically thin in $H_{1 .}$. Because the northern auroral oval is offset from the rotational pole, the effect of the slant path brightening is reduced somewhat by smearing during the exposure. During a typical exposure of $15 \mathrm{~min}$, the planet rotates by about $9^{\prime \prime}$ of longitude. The slant path enthancement is also reduced if the actual au. roral structure is unresolved by the FOC. A resolution of about 0.1 arc sec implies that structures smaller than about $400 \mathrm{~km}$ are diluted. For comparison, the scale height near the homopause on Jupiter is likely to be about $100 \mathrm{~km}$ or 50 and a degree of latitude at $60 \mathrm{~N}$ subtends about $600 \mathrm{~km}$. We assumed that the aurora's vertical emission rate can be represented by a Chaoman profile, used the nominal value of $100 \mathrm{~km}$ for the atmospheric scale height and an auroral height of $500 \mathrm{~km}$ above the cloud tops, and accounted for the dilution caused by the spatial resolution of the FOC but not for smearing from the rotation of Jupiter).

12. J. C. Gérard, V. Dols. R. Prangé, F. Paresce, Planet. Space Sci. in press

13. J. Caldwell, B. Turgeon, X.-M. Hua, Science 257 $1512(1992)$

14. This value can be considered as a lower limit be cause the aberrated point spread function of the telescope and the blurring effect of the planetary rotation spreads the light of a point source over many pixels and decreases the apparent emission rate of localized emission. A rough estimate of this effect may be obtained trom the deconvolved (Wiener) version of Fig. 2A, although this method does not necessarily provide correct photometric values. The brightest region in this case reaches $\cdots 10 \mathrm{MF}$ of $\mathrm{H}$. Lyman emission.

15. J. C. Gerard and V. Singh, J. Geophys. Res. 87. 4525 (1982).

16. H. J. Waite et al., ibid. $88,6143(1983)$

17. D. Rego. R. Prangé, J. C. Gérard, ibid. 99, 17075 (1994)

18. The results of the $1 \mathrm{~W} \mathrm{~m}$ "' aurora excited by electrons with the Ulysses energy spectrum imply an $\mathrm{H},{ }^{\prime}$ production rate exceeding $2 \times 10^{4} \mathrm{~cm}$ " with a peak production expected near the 1 - $\mu$ bar pressure level and a peak $\mathrm{H}$, density on the order of $10^{\mathrm{f}}$ $\mathrm{cm}$ :" The corresponding heating rate also peaks at this pressure with a vertically integrated heat flux of over 450 ergs $\mathrm{cm}$ '' $\mathrm{s}$ 1. The heating rate profile calculated from the modef can be combined with recent cooling rate calculations (scaled so that the vertically integrated cooling rate equals the vertically integraled heating rate and peaks at a pressure level of $1 \mu \mathrm{bar}$, just above the methane homopause, that is, cool-to-space approximation. These values were used as inputs in a $1 \mathrm{D}$ thermal conduction equation (26) to estimate the auroral thermal profile.

19. L. M. Tratton, J. C. Gerard, G. Munhoven, J. H Waite. Astrophys. J. 421,816 (1994)

20. P. Drossant et al., J. Geophy/s. Res. 98, 18803 1993).

21. J. T. Clarke et at. Astrophys. J. Lett 430,73(1994)
22. G. R. Gladstone and T. Skinner, NASA SP-494 (1989), pp. 221-228.

23. M. Hammond and $\mathrm{d}$. Gosling, personal communication.

24. M. K. Dougherty, D. J. Southwood, A. Balogh, E. J. Smith. Planet. Space Sci. 41, 291 (1993); R. Prangé, M. Dougherty, M. Dunlop, A. Balogh, V. Dols, Eos 74, $43(1993)$.

25. V. Vasyliunas, Geophys. Res. Lett. 21, 401 (1994)

26. H. J. Waite, M. L. Chandler, R. V. Yelle, B. R. Sandel, T. E. Cravens, J. Geophys. Res. 93, 14295 (1988).

27. E. P Connerney, in Planetany Radio Emission III. $\mathrm{H}$ Rucker, M. L. Kaiser, S. J. Bauer, Eds. (Austrian Academy ol Sciences Press, Vienna, 1992), pp. 1333.

28. JCG acknowledges support from the Belgian National Fund for Scientific Research (FNPS) and from the Belgian Federal Science Policy Office, Prime Minister's Services. RP is grateful to J. Gos ling, M. Hammond, K. Maeda, H. Oya, and P. Zarka for fruitful discussions and for having kindly provided unpublished data on the Jovian radio emission and on the solar wind. She acknowledges partial suppor of this study by grant INSU-PNP/80374200. J.H.W acknowledges support from Space Telescope Science institute grant GO-4608.02-92A and Nationa Aeronautics and Space Administration (NASA) Planetary Atmosphere grant NAGW-3624. This study is based on observations with the NASA-ESA HST that were obtained at the Space Telescope Science in stitute, which is operated by AURA for NASA under contract NAS5-26555

11 July 1994; accepted 27 September 1994 